\title{
Microbiological spectrum of pneumonia in patients with lung cancer
}

\author{
Tomohiro Tamura, Hiroaki Satoh
}

Division of Respiratory Medicine, Mito Medical Center, University of Tsukuba, Tsukuba, Japan

Contemp Oncol (Pozn) 2016; 20 (3): 266-266 DOI: $10.5114 /$ wo.2016.61570

We read with interest the article entitled "Retrospective analysis of the microbiological spectrum of pneumonia in Turkish patients with lung cancer" by Avci et al. (Contemporary Oncology 2016; 20: 63-66). We are surprised to read that the most frequent isolate from sputum of lung cancer patients with pneumonia was Aspergillus fumigatus [1]. We do understand gram negative bacteria such as Haemophillus influenza and Pseudomonas aeruginosa cause pneumonia in lung cancer patients, but we do not encounter fungal pneumonia in lung cancer patients with usual platinum-based chemotherapy. With regards to the results, we do appreciate hearing from the authors whether lung cancer patients with Aspergillus fumigatus in their sputum had had antimicrobial chemotherapy frequently. For the most important concern, we do wonder whether Aspergillus fumigatus truly caused pneumonia, and whether the antifungal therapy was required for the fungal pneumonia. We do understand the most frequent isolate from sputum was Aspergillus fumigates, however, we do wonder whether it would cause pneumonia. Were there any possibilities of contamination or colonization? We would like to ask the authors what was the definition of "cause of pneumonia" from the bacteriological and clinical point of view.

\section{References}

1. Avcı N, Hartavi M, Kaçan T, et al. Retrospective analysis of the microbiological spectrum of pneumonia in Turkish patients with lung cancer. Contemp Oncol (Pozn) 2016; 20: 63-6.

\section{Address for correspondence}

Hiroaki Satoh MD, PhD

Division of Respiratory Medicine

Mito Medical Center

University of Tsukuba, Miya-machi 3-2-7

Mito, Ibaraki, 310-0015, Japan

tel. +81-29-231-2371

e-mail: hirosato@md.tsukuba.ac.jp

Submitted: 15.05 .2016

Accepted: 22.06 .2016 